\title{
Morphology and scaling in the noisy Burgers equation Soliton approach to the strong coupling fixed point
}

\author{
Hans C. Fogedby \\ * Institute of Physics and Astronomy, University of Aarhus, DK-8000, Aarhus C, Denmark \\ and \\ NORDITA, Blegdamsvej 17, DK-2100, Copenhagen Ø, Denmark
}

(March 30, 2017)

\begin{abstract}
The morphology and scaling properties of the noisy Burgers equation in one dimension are treated by means of a nonlinear soliton approach based on the Martin-Siggia-Rose technique. In a canonical formulation the strong coupling fixed point is accessed by means of a principle of least action in the asymptotic nonperturbative weak noise limit. The strong coupling scaling behaviour and the growth morphology are described by a gas of nonlinear soliton modes with a gapless dispersion law and a superposed gas of linear diffusive modes with a gap. The dynamic exponent is determined by the gapless soliton dispersion law, whereas the roughness exponent and a heuristic expression for the scaling function are given by the form factor in a spectral representation of the interface slope correlation function. The scaling function has the form of a Lévy flight distribution.
\end{abstract}

PACS numbers: 05.40.+j, 05.60.+w, 75.10.Jm

There is a current interest in the scaling properties and general morphology of nonequlibrium models. Here the noisy Burgers equation in one dimension provides maybe the simplest continuum description of an open driven nonlinear system [1] [) This equation has the form of a conserved Langevin equation

$$
\partial u / \partial t=\nu \nabla^{2} u+\lambda u \nabla u+\nabla \eta,
$$

where $\nu$ is a damping constant characterizing the linear diffusive term, $\lambda$ a coupling strength for the nonlinear mode coupling term, and $\eta$ a Gaussian white noise driving the system into a stationary state and correlated according to

$$
\left\langle\eta(x t) \eta\left(x^{\prime} t^{\prime}\right)\right\rangle=\Delta \delta\left(x-x^{\prime}\right) \delta\left(t-t^{\prime}\right) .
$$

The equation (11) has been discussed extensively in particular as a model for the stochastic dynamics of the slope field $u=\nabla h$ for the self-affine growth of an interface subject to annealed noise from the drive or environment described by the Kardar-Parisi-Zhang (KPZ) equation

$$
\partial h / \partial t=\nu \nabla^{2} h+(1 / 2) \lambda(\nabla h)^{2}+\eta .
$$

The main emphasis has been on the scaling properties of the slope field in the large $x$ - long $t$ limit as embodied in the dynamical scaling form [3, 4 ,

$$
\left\langle u(x t) u\left(x^{\prime} t^{\prime}\right)\right\rangle=\left|x-x^{\prime}\right|^{-2(1-\zeta)} f\left(\left|t-t^{\prime}\right| /\left|x-x^{\prime}\right|^{z}\right),
$$

where $\zeta$ is the roughness exponent, $z$ the dynamic exponent, and $f$ the scaling function; $f(w) \propto w^{-2(1-\zeta) / z}$ for large values of its argument. In this context the nonlinear Galilean symmetry of (11)

$$
x \rightarrow x-\lambda u_{0} t, \quad u \rightarrow u+u_{0}
$$

with $\lambda$ entering as a structural constant implies the scaling law

$$
\zeta+z=2 .
$$

Another property specific to one dimension is the stationary probability distribution [2, 何,

$$
P(u) \propto \exp \left[-(\nu / \Delta) \int d x u^{2}\right] .
$$

independent of $\lambda$. Hence $u$ is an independent Gaussian variable, $\left\langle u(x) u\left(x^{\prime}\right)\right\rangle=(\Delta / 2 \nu) \delta\left(x-x^{\prime}\right)$, and the height field $h=\int d x u$ performs random walk with exponent $\zeta=1 / 2$. The scaling law (6) then yields $z=3 / 2$, defining the Burgers-KPZ universality class. This result also follows from dynamic renormalization group calculations [3, 14 but the associated infrared stable strong coupling fixed point does not seem accessible by the methods of renormalized perturbation theory and the $\epsilon$-expansion.

In the linear Edwards-Wilkinson (EW) case for $\lambda=0$ the slope correlations are

$$
\langle u(k \omega) u(-k-\omega)\rangle=\frac{\Delta k^{2}}{\omega^{2}+\left(\nu k^{2}\right)^{2}},
$$

yielding the exponents $\zeta=1 / 2$ and $z=2$, characterizing the EW universality class with scaling function

*Permanent address 


$$
f(w)=(\Delta / 2 \nu)(4 \pi \nu)^{-1 / 2} w^{-1 / 2} \exp [-1 / 4 \nu w] .
$$

In a recent letter [5] we approached the strong coupling fixed point behaviour by means of a mapping of the Burgers equation (11) onto a solid-on-solid model and further onto a discrete spin $1 / 2$ chain model. In a harmonic oscillator representation valid for large spin combined with a continuum limit this approach eventually yields a Hamiltonian description and a set of coupled equations of motion with soliton solutions with dispersion $E \propto p^{3 / 2}$, yielding the exponent $z=3 / 2$, characterizing the strong coupling fixed point. The approach furthermore gave a tentative picture of a growing interface in terms of a soliton gas of paired solitons representing a growing step in the height field.

Here we present a unified approach to the noisy Burgers equation based on a Martin-Siggia-Rose path integral. This method provides a generalization of the stationary distribution (77) to the time-dependent nonlinear case and yields the following physical picture and scaling results: 1) In the weak noise limit the general morphology of a growing interface in the stationary regime is described by a Landau-type quasi-particle many-body theory in terms of a gas of nonlinear soliton modes with superposed linear diffusive modes.

2) The noise-driven fluctuations correspond to "quantum fluctuations" in the underlying non-Hermitian relaxational "quantum description", yielding the quasiparticle picture.

3) The scaling exponents and the scaling function follow as a by-product from the soliton dispersion law and the spectral representation of the correlations. The manybody formulation explains the robustness of the roughness exponent under a change of universality class.

From a field theoretical point of view we identify the noise strength $\Delta$ in (2) as the effective small parameter. Furthermore, the fundamental probability distribution or path integral has an essential singularity for $\Delta=0$. Hence our approach is based on a nonperturbative saddle point or steepest descent approximation to the path integral. It is precisely in this respect that the dynamic renormalization group method based on an expansion in $\lambda$ and in the noise contraction $\Delta$ fails to access the strong coupling fixed point.

The special role of the noise is easy to understand. The turning on of the noise in the Burgers equation is a singular process in the sense that even weak noise $\Delta \sim 0$ will eventually drive the system into a stationary state whereas for $\Delta=0$ the noiseless deterministic Burgers equation (assuming vanishing currents at the boundaries) describes a damped interface, although with pattern formation or dissipative structures formed by the nonlinear cascade term in the transient time regime. In the presence of noise the system passes from the transient to the stationary regime at a crossover time $t_{c o}$ determined by the noise strength. In the linear EW case $t_{c o}$ is of or- der $1 /\left(\nu k^{2}\right) \log (1 / \Delta)$ showing that $t_{c o} \rightarrow \infty$ for $\Delta \rightarrow 0$; $k$ is the wave number of the particular mode considered (note that $t_{c o} \rightarrow \infty$ also for $k \rightarrow 0$, characteristic of a conserved hydrodynamical mode). In the linear EW case the singular character of the noise strength $\Delta$ is reflected in the stationary distribution (7) which has an essential singularity for $\Delta=0$.

It is well-known that the noiseless Burgers equation for $\Delta=0$ [1, 过, 6], $\partial u / \partial t=\nu \nabla^{2} u+\lambda u \nabla u$, supports paritybreaking right hand solitons connected by ramp solutions, corresponding to cusps connected by convex parabolic segments in the height field. Superposed on the solitons are linear diffusive modes with a gap in the spectrum. This configuration constitutes the growth pattern in the transient time regime. For $\lambda=0$ the solitons disappear and the equation reduces to the linear diffusion equations supporting gapless diffusive modes.

In the stationary noise-driven case for $\Delta \neq 0$ the soliton excitations and diffusive modes also play an important role in the analysis of the physics and scaling behaviour of the strong coupling fixed. In the noisy case the solitons appear as stationary or saddle point solutions to the path integral in the weak noise limit; the diffusive modes correspond to Gaussian fluctuations about the stationary points. Also the noise fluctuations lift the broken parity symmetry and excites both right and left hand solitons. As regards the diffusive modes both decaying and growing modes are excited in accordance with the time reversal invariance of the stationary regime as for example reflected in the evenness in $\omega$ of the slope correlations (8) in the linear case.

In terms of the Martin-Siggia-Rose path integral [7] the slope correlations are given by [8]

$$
\left\langle u(x t) u\left(x^{\prime} t^{\prime}\right)\right\rangle=\frac{\int \prod_{x t} d u d \varphi \exp [i(\nu / \Delta) S] u(x t) u\left(x^{\prime} t^{\prime}\right)}{\int \prod_{x t} d u d \varphi \exp [i(\nu / \Delta) S]}
$$

which provides the generalization of the stationary EW case $\left\langle u(x) u\left(x^{\prime}\right)\right\rangle=\int \prod_{x} d u P(u) u(x) u\left(x^{\prime}\right)$ with $P(u)$ given by (耳). The action $S$ in (10) has the form

$$
S=\int d x d t[u d \varphi / d t-\mathcal{H}(u, \varphi)]
$$

with Hamiltonian density

$$
\mathcal{H}=-i(\nu / 2)\left[(\nabla u)^{2}+(\nabla \varphi)^{2}\right]+(\lambda / 2) u^{2} \nabla \varphi .
$$

The correspondence of $10,11,12$ with the Feynman phase space path integral [9] allows us to consider $\Delta / \nu$ as an effective Planck constant and the slope field $u$ and noise field $\varphi$ as canonically conjugate variables satisfying the Poisson bracket $\left\{u(x), \varphi\left(x^{\prime}\right)\right\}=\delta\left(x-x^{\prime}\right)$. It is also characteristic of a stochastic growth problem that the Hamiltonian, being the generator in the relaxational master equation, in general is complex. The imaginary 
harmonic part drives the diffusive modes whereas the real nonlinear term describes the intrinsic growth of the interface. The path integral replacing the noisy Burgers equation is deterministic, the noise-driven fluctuations being replaced by the contributing paths or configurations in the path integral.

The canonical form of the path integral allows for $a$ principle of least action. In other words, in the weak noise limit $\Delta \rightarrow 0$ we can determine "classical" solutions or orbits from the stationary points of the action. The weak noise solutions are then given by the equations of motion, $d u / d t=\{\mathcal{H}, u\}=-\delta \mathcal{H} / \delta \varphi$ and $d \varphi / d t=\{\mathcal{H}, \varphi\}=\delta \mathcal{H} / \delta u$,

$$
\frac{\partial u}{\partial t}=-i \nu \nabla^{2} \varphi+\lambda u \nabla u, \quad \frac{\partial \varphi}{\partial t}=+i \nu \nabla^{2} u+\lambda u \nabla \varphi
$$

The deterministic nonlinear coupled field equations (13) for the slope and noise fields thus replace the noisy Burgers equation (1) in the asymptotic nonperturbative weak noise limit. We note that i) the equations are invariant under a shift of $\varphi$ reflecting the conserved noise in (11) and ii) like the noisy Burgers equation, invariant under the Galilean transformation (5) supplemented with $\varphi \rightarrow \varphi$.

In addition to the constant solutions $(u, \varphi)=\left(u_{0}, \varphi_{0}\right)$, corresponding to the zero-energy stationary state, the field equations also support nonlinear soliton solutions of both parities. The static solitons are given by

$$
u= \pm u_{+} \tanh \left(k_{s}\left(x-x_{0}\right)\right), \quad k_{s}=\lambda u_{+} / 2 \nu .
$$

Here $k_{s}$ is a wavenumber depending on the soliton amplitude, setting the inverse length scale, and $x_{0}$ a center of mass position. Note that only the right hand soliton appears in the noiseless case. Boosting the soliton to a finite velocity $v$ and denoting the boundary values by $u_{ \pm}$ the general soliton condition is [5]

$$
u_{+}+u_{-}=-2 v / \lambda
$$

relating the soliton amplitude and off-set to the propagation velocity.

For $\lambda=0$ the field equations yield diffusive modes $u=A \exp (-i \omega t+i k x)+$ c.c. with gapless dispersion

$$
\omega=-i \nu k^{2}
$$

The Hamiltonian (12) is harmonic, the path integral (10) Gaussian, and it is an easy task to evaluate the slope correlations (8).

It is a well-known feature of field theoretical saddle point soliton or instanton calculations that multi-soliton solutions also contribute to the stationary points [9]. Here this implies that the extremal path in the weak noise limit corresponds to a dilute soliton gas of right and left hand solitons matched according to the soliton condition
(15). In other words, the path integral formulation directly produces a physical picture of the morphology of a growing interface. The natural variable is the slope field $u$. A single soliton configuration connecting two stationary slope configuration corresponds to the propagating top or bottom of a growing step in the height field $h$. A single moving step in $h$ is represented by a pair of solitons matched according to (15). In a similar fashion a moving plateau in $h$ corresponds to four solitons, a growing tip to a three-soliton configuration, etc. The morphology of a growing interface can thus be interpreted as a dilute gas of solitons corresponding to moving steps in the height field. We note that the noise radically changes the morphology from the noiseless transient case in that the noise excites both "up" and "down" cusps in $h$. Superposed on the soliton gas are linear diffusive "ripple" modes with a gap in the spectrum.

The canonical formulation of the path integral enables us to associate energy, momentum, and action with a given soliton configuration. This allows for a dynamical selection criterion similar to the Boltzmann factor $\exp (-E / T)$ in equilibrium statistical mechanics which associates an energy $E$ with a given configuration contributing to the partition function; in the dynamical case the action $S$ provides the weight function for the dynamical configuration.

The energy is given by $E=\int d x \mathcal{H}$ whereas the momentum follows from the Poisson bracket algebra, $P=\int d x u \nabla \varphi$. For a single soliton we have in terms of the boundary values, $E= \pm i(\lambda / 6)\left(u_{+}^{3}-u_{-}^{3}\right)$ and $P= \pm i(1 / 2)\left(u_{+}^{2}-u_{-}^{2}\right)$. In the case of a single moving soliton satisfying $u_{-}=0$ we obtain in particular

$$
E=(4 i / 3)|v|^{3} / \lambda^{2}, \quad P=-(2 i) v|v| / \lambda^{2} .
$$

We note the nonlinear velocity dependence characteristic of soliton solutions [10,11. Eliminating $v$ we obtain the soliton dispersion law $E=\lambda(\sqrt{2} / 3) \exp (i \theta) P^{3 / 2}$, where the phase $\theta=\pi+(\pi / 4)(v /|v|)$.

The linear diffusive mode spectrum in the presence of the solitons is analyzed by a linear stability analysis of the field equations (13). Like in the noiseless case 6 the associated eigenvalue problem is exactly soluble 12. In addition to zero-eigenvalue bound states corresponding to the soliton translation modes lifting the broken translational symmetry, the spectrum also has a band of phase shifted diffusive modes with a gap in the dispersion law

$$
\omega=-i \nu\left(k^{2}+k_{s}^{2}\right)
$$

depending on the soliton amplitude according to (14).

In order to complete the analysis of the noisy Burgers equation it remains to discuss the contribution of the Gaussian fluctuations in the path integral about the solitons. The basic information lies in the path integral and will be discussed elsewhere. Here we take a heuristic point of view and discuss the fluctuations in terms 
of the underlying non-Hermitian relaxational "quantum field theory" leading to the path integral by the usual Feynman procedure [9]. The canonical fields $u$ and $\varphi$ must now be interpreted as "quantum operators" satisfying a commutator algebra, the Hamiltonian $(12)$ is a nonHermitian operator, and the field equations (13) are the associated Heisenberg equations of motion. The "classical" localized soliton solution (14) becomes a delocalized "quantum" quasi-particle with frequency $\Omega=E /(\Delta / \nu)$ and wavenumber $K=P /(\Delta / \nu)$. For a pair of matched quasi-particles representing a growing step the "quantum" dispersion law is

$$
\Omega=\lambda(1 / 3)(\Delta / \nu)^{1 / 2} e^{i \theta} K^{\frac{3}{2}} .
$$

Evaluating the group velocity of a wave packet representing the "quantum soliton" we find the classical propagation velocity, in complete accordance with an effective correspondence principle. In a similar way the linear diffusive modes are "quantised" becoming quasi-particles corresponding to "ripple" modes on the solitons with dispersion law (18) 10.

The picture of the growing interface is thus complete. The noise-induced fluctuations admits a "quantum mechanical" interpretation. The dominant morphology consists of a fluctuating "quantum" gas of solitons with a gapless dispersion law with exponent $3 / 2$. Superposed on the soliton gas are linear diffusive "ripples" modes with a gap in the dispersion depending on the soliton amplitude. In the limit of vanishing growth for $\lambda \rightarrow 0$ the solitons disappear and the diffusive modes become gapless corresponding to thermodynamic fluctuations governed by the EW equation with Boltzmann weight (7).

In the "quantum interpretation" the slope correlation function $\langle u(x t) u(00)\rangle$ is given by a time-ordered Green's function $\langle 0|T \hat{u}(x t) \hat{u}(00)| 0\rangle[9]$. Here $\hat{u}$ is the time-dependent "quantum" slope field and $|0\rangle$ denotes the appropriate stationary zero-energy state. Assuming $t>0$, using the Hamiltonian and momentum operators to "displace" the field at $(x, t)$ to $(0,0)$, and inserting a complete set of momentum "quasi-particle" states, we derive an effective spectral representation which allows us to discuss the scaling properties in simple terms,

$$
\langle u(x t) u(00)\rangle=\int d K G(K) e^{-i(\Omega t+K x)} .
$$

Here $G(K)$ is an effective form factor which in general is complex owing to the non-Hermitian Hamiltonian (12). At long distances (20) samples the small wave number region in the integral and assuming that the form factor is regular for small $K$ and a general dispersion law of the form

$$
\Omega=\tilde{\Delta}+K^{\mu}
$$

characterized by the gap $\tilde{\Delta}$ and the exponent $\mu$, we obtain, absorbing $x$ in a rescaling of $K$

$$
\langle u(x, t) u(0,0)\rangle \propto e^{-i \tilde{\Delta} t} x^{-1} \int d K e^{-i K^{\mu}\left(t / x^{\mu}\right)-i K} .
$$

First, in the presence of a gap there is no scaling due to the exponential prefactor in (22), consequently, only gapless excitations contribute to the scaling behaviour. For $\tilde{\Delta}=0$ comparing (22) with (4) we infer $z=\mu$ and $\zeta=1 / 2$.

In the linear EW case the diffusive gapless modes with dispersion (16) exhaust the spectrum and we obtain $\mu=z=2$, corresponding to the EW universality class. The spectral form (22) yields the scaling function in (9).

In the nonlinear Burgers-KPZ case the nonlinear soliton modes with gapless dispersion (19) exhaust the bottom of the spectrum and yields $\mu=z=3 / 2$ for the Burgers-KPZ universality class. The linear diffusive modes develop a gap, become sub-dominant and do not contribute to the scaling. This mechanism explains the change in universality class: The universality class is determined by the dominant gapless excitation. The spectral form (20) also elucidates the robustness of the exponent $\zeta$ which is the same for both universality classes. For the stationary fluctuations we set $t=0$ in 20 and the resulting scaling function yielding $\zeta$ does not depend on the specific quasi-particle dispersion law.

Finally, we also obtain a heuristic expression for the scaling function

$$
f(w)=\int d K e^{-i\left(K^{z} w+K\right)}
$$

which incidentally has the same form as the probability distribution for Lévy flights [13].

In this letter we have presented the outline of a novel approach to the growth morphology and scaling behaviour of the noisy Burgers equation in one dimension; details will appear elsewhere. Using the Martin-SiggiaRose technique in a canonical form we have demonstrated that the so far elusive strong coupling fixed point behaviour is associated with an essential singularity in the noise strength and can be accessed by appropriate field theoretical soliton techniques.

Discussions with J. Krug, M. Kosterlitz and A. Svane are gratefully acknowledged.

[1] J.M. Burgers, The Nonlinear Diffusion Equation, (Riedel, Dordrecht, 1974)

[2] J. Krug, Adv. Phys. 46, 139 (1997)

[3] D. Forster, D.R. Nelson, and M.J. Stephen, Phys. Rev. Lett. 36, 867 (1976); Phys. Rev. A 16, 732 (1977) 
[4] M. Kardar, G. Parisi, and Y.C. Zhang, Phys. Rev. Lett. 56, 889 (1986); E. Medina, T. Hwa, M. Kardar, and Y.C. Zhang, Phys. Rev. A 39, 3053 (1989).

[5] H.C. Fogedby, A.B. Eriksson and L.V. Mikheev, Phys. Rev. Lett. 75, 1883 (1995)

[6] H.C. Fogedby, cond-mat/9709058

[7] P.C. Martin, E.D. Siggia, and H.A. Rose, Phys. Rev. A 8, 423 (1973); R. Baussch, H.K. Janssen, and H. Wagner, Z. Phys. B 24, 113 (1976)

[8] In order to obtain the canonical form we have shifted the noise field as in [5].

[9] A. Das, Field Theory - A Path Integral Approach (World Scientific, Singapore 1993)

[10] H.C. Fogedby, Z. Phys.B 41, 115 (1981)

[11] $E$ and $P$ can be made real by rotating the noise field, $\varphi \rightarrow i \varphi$ in (10).

[12] H.C. Fogedby, P. Hedegaard and A. Svane, Physica 132B, 17 (1985)

[13] H.C. Fogedby, Phys. Rev. Lett. 73,2517 (1994) 